\title{
Low Strength of Correlation between the Intensity of Neutrophil Elastase Expression in Lesional Skin and the Level of Serum IgA Antibodies to Epidermal Transglutaminase in Dermatitis Herpetiformis
}

\author{
M. Bowszyc-Dmochowska ${ }^{1, *}$, A. Seraszek ${ }^{2}$, E. Kaczmarek ${ }^{2}$, J. Gornowicz $^{1}$ and M. Dmochowski ${ }^{1}$ \\ ${ }^{1}$ Department of Dermatology and ${ }^{2}$ Department of Pathology, University School of Medicine, Poznań, Poland
}

\begin{abstract}
Whereas it has been shown that neutrophil elastase (NE) is a crucial enzyme degrading the dermal-epidermal junction (DEJ) in bullous pemphigoid (BP), experimental studies on the role of NE in dermatitis herpetiformis (DH), a disease in which, as in BP, an intra-lamina lucida blister is formed, are scanty. The aim of this study was to analyse whether there is a correlation between levels of serum IgA antibodies to the epidermal transglutaminase (TG3), an enzyme believed to be the autoantigen of $\mathrm{DH}$, and expression of $\mathrm{NE}$ in lesional skin in DH. A series of 21 consecutive patients with DH was studied. The levels of IgA antibodies to TG3 in sera were calculated with ELISA. The expressions of NE were examined with immunohistochemical technique in sections of lesional skin using a mouse monoclonal antibody to human NE. The digital microscopic image analysis with the appropriate software was then used to measure intensities of $\mathrm{NE}$ expression. The correlation between the intensity of NE expression in lesional skin and the level of serum IgA antibodies to TG3 in DH was of low strength. Thus, it is speculated that in DH the engagement of IgA autoantibodies to the enzyme, TG3, on cutaneous neutrophils might not be a principal stimulus to releasing NE, the enzyme known to degrade DEJ in subepidermal blistering diseases with autoimmunity to DEJ structural proteins.
\end{abstract}

\section{REPORT}

The plausible pathophysiology of dermatitis herpetifomis $(\mathrm{DH})$ is that the $\mathrm{Fc}$ region of IgA autoantibody to epidermal transglutaminase (TG3), an enzyme believed to be the most disease-specific autoantigen of $\mathrm{DH},[1]$ activates neutrophils by engaging their CD89 receptor which leads to the release of neutrophil elastase (NE), NE-mediated destruction of the dermal-epidermal junction (DEJ) and the formation of an intra-lamina lucida blister. Whereas it was shown that NE is crucial DEJ-degrading enzyme in bullous pemphigoid (BP), [2-3] experimental studies on the role of $\mathrm{NE}$ in $\mathrm{DH}$ are scanty. Early data suggested that in blister fluids in patients with DH an activity of elastase, probably NE, can be detected [4-5]. The aim of this study was to analyse whether there is a correlation between levels of serum IgA antibodies to TG3 and expression of NE in lesional skin in DH.

NE deposits and IgA antibodies to TG3 were evaluated in a series of 21 consecutive dapsone-untreated patients with DH, regardless whether they had concomitant intestinal problems or not. The diagnosis of DH in each case was made when whichever pattern of possible 7 diagnostic patterns of granular IgA deposition was detected with direct immunofluorescence of nonlesional skin [6-8]. The levels of IgA antibodies to TG3 in sera were calculated with ELISA in $\mathrm{AU} / \mathrm{ml}$ (manufacturer's cut-off value 18AU/ml) (Immundiagnostic, Germany). The expressions of NE were examined with immunohistochemical technique in sections of lesional

*Address correspondence to this author at the Department of Dermatology, University School of Medicine, Poznań, Poland;

E-mail: dmoch@sylaba.poznan.pl skin showing histological features of $\mathrm{DH}$, namely neutrophilrich infiltrate at the tips of dermal papillae, using a mouse monoclonal antibody to human NE (Dako, Denmark) and LSAB + system+ HRP visualisation kit (Dako, Denmark). The digital microscopic image analysis with the appropriate software [9] was then used to measure intensities of NE expression in the area of the visibly strongest immunohistochemical reaction under the magnification x 200 in percentages of expression [10]. The results of NE expression analysis in a representative DH patient are shown on Figs. (1-4). The appropriate immunohistochemical procedure controls were performed. Statistical analysis was done using Spearman's rank correlation coefficient.

The strength of correlation between the intensity of NE expression in lesional skin and the level of serum IgA antibodies to TG3 in DH (Spearman's $r=0.2822 ; P$-value $<0.05$ ) (Fig. 5) was low and should not be regarded as a moderate relationship [11]. Eleven DH cases had the level of serum IgA antibodies to TG3 above the manufacturer's cut-off value (52.4\%); range of levels was 4.79-176.52 AU/ml. The low percentage of DH cases with elevated levels of serum IgA antibodies to TG3 in this study should not be considered unusual since in a recently published investigation only $52 \%$ of DH patients were shown to have those levels elevated [12]. It is known that dapsone impairs the functions of neutrophils, but our results were not influenced by this drug as none of patients was treated with dapsone prior to the establishment of the diagnosis of DH. The reliable information on the use of gluten-free diet by patients studied was unfortunately unavailable to us. It is conceivable that the strict gluten-free diet should normalize serum levels of IgA antibodies 


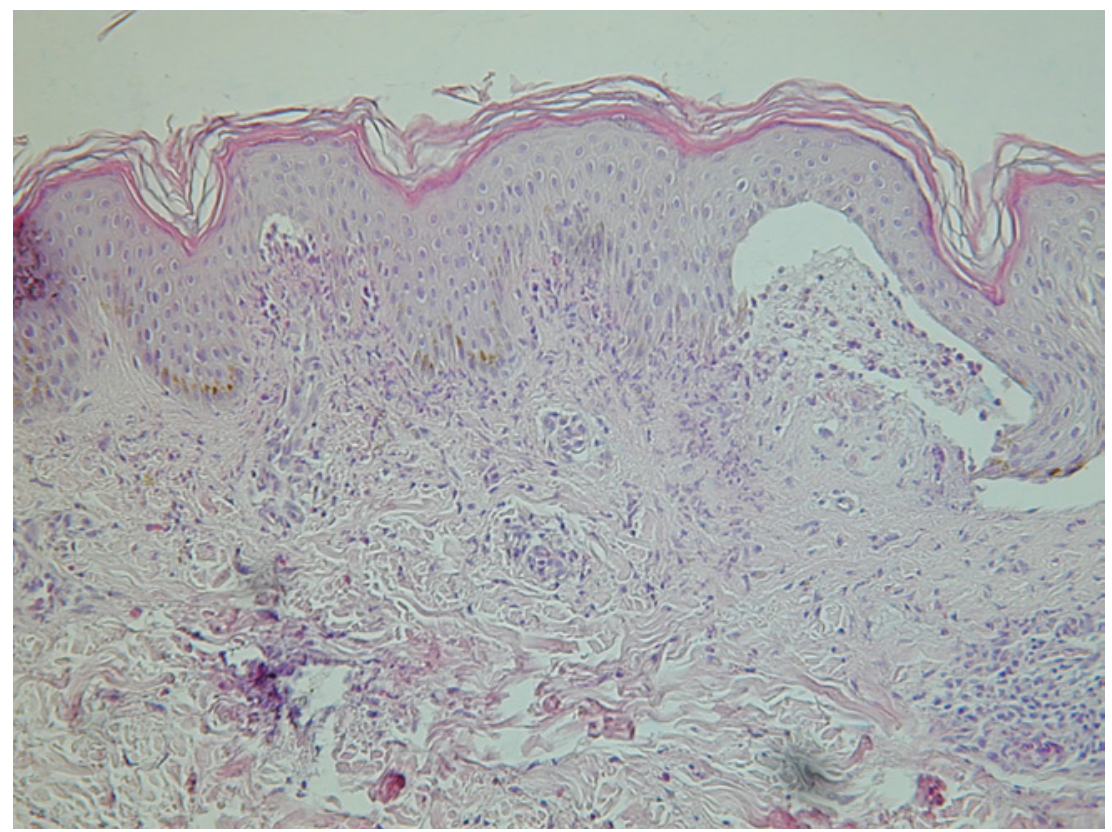

Fig. (1). Neutrophil-rich infiltration at the tips of dermal papillae in a patient with DH (H+E staining) (magnification, x200).

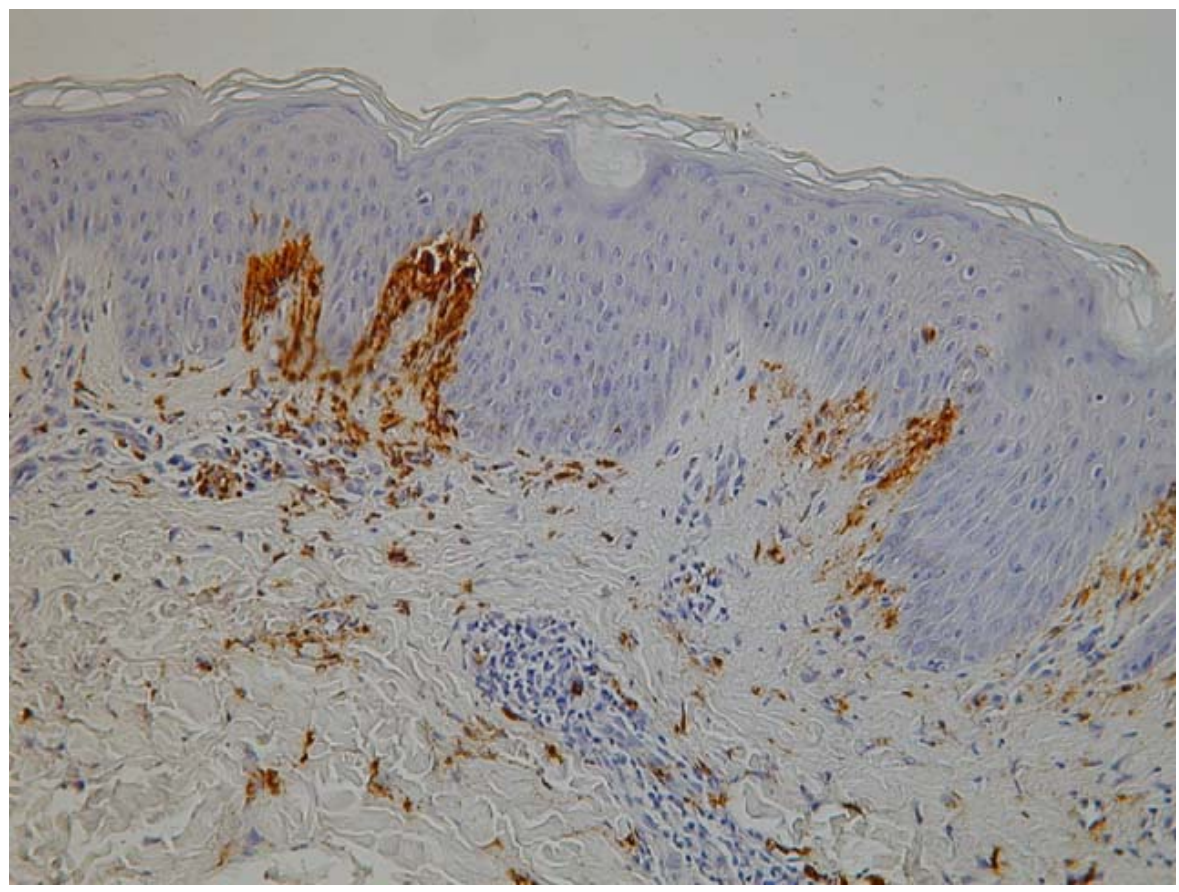

Fig. (2). NE deposits in immunohistochemistry in lesional skin in a patient with DH (magnification, x200).

not only to tissue transglutaminase (TG2) but also to TG3, so our results could be influenced by a dietary factor. Still, all our patients had cutaneous rash severe enough to prompt them to seek dermatological attention, so we believe that their dietary habits had low impact on their skin disease. Therefore, we feel that levels of $\operatorname{IgA}$ antibodies to TG3 obtained in our study truly reflect the severity of their skin disease, irrespectively of either intestinal or dietary considerations.

One might even speculate, on the basis of our study, that in DH the engagement of $\operatorname{IgA}$ autoantibodies to the enzyme, TG3, on cutaneous neutrophils might not be a principal stimulus to releasing NE, an enzyme known to degrade DEJ in subepidermal blistering diseases with autoimmunity to DEJ structural proteins [2]. This hypothesis should be experimentally verified, especially as we have previously shown that the expressions of NE in lesional skin in patients with DH and BP are not statistically different [10]. Still, there is a possibility that this stimulus is provided not by pooled $\operatorname{IgA}$ autoantibodies to TG3, but specifically by $\operatorname{IgA} 1$ or even IgA2 subclass autoantibodies to TG3. As 2 splicing isoforms of human TG3 have recently been identified, [13] it is currently unclear whether the $\operatorname{IgA}$ autoantibodies to those TG3 isoforms might differ in neutrophil activating capacities, hence whether there might be unequal NE releasing in 


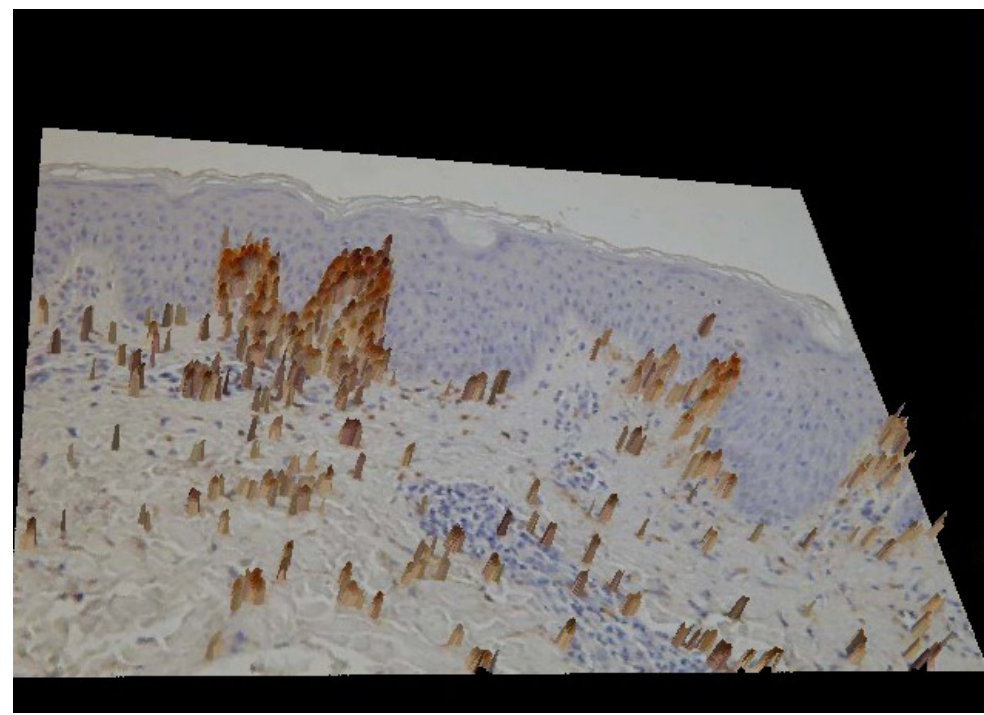

Fig. (3). Intensity of NE deposits processed with digital microscopic image analysis superimposed on NE deposits immunohistochemistry in lesional skin in a patient with $\mathrm{DH}$.

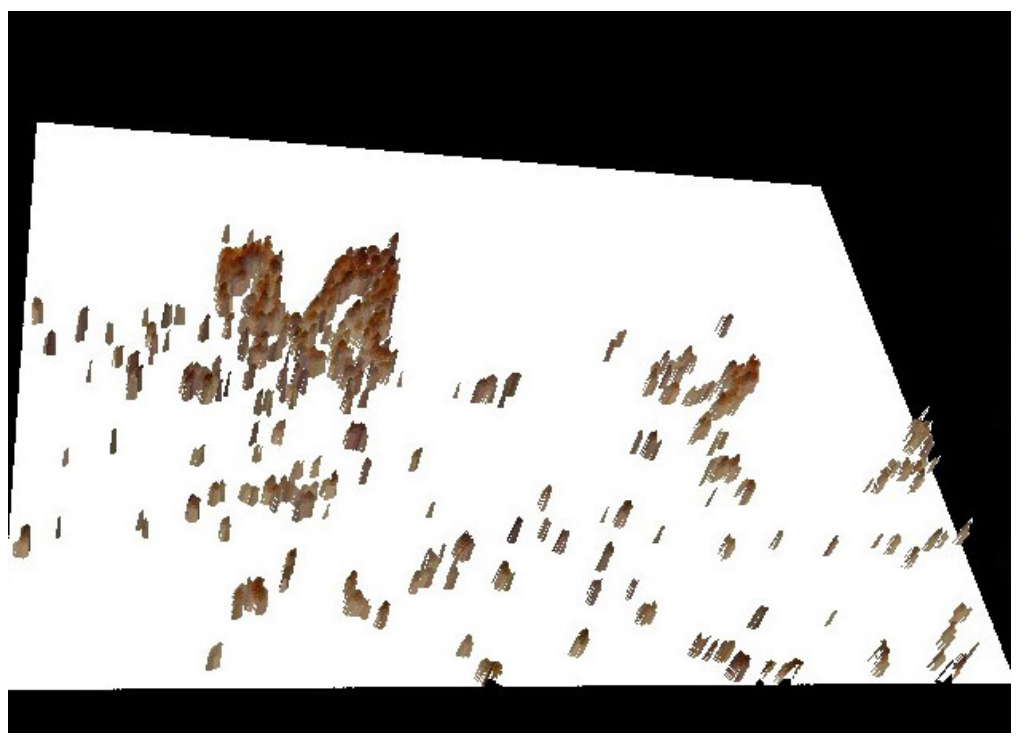

Fig. (4). Intensity of NE deposits processed with digital microscopic image analysis in lesional skin in a patient with DH.

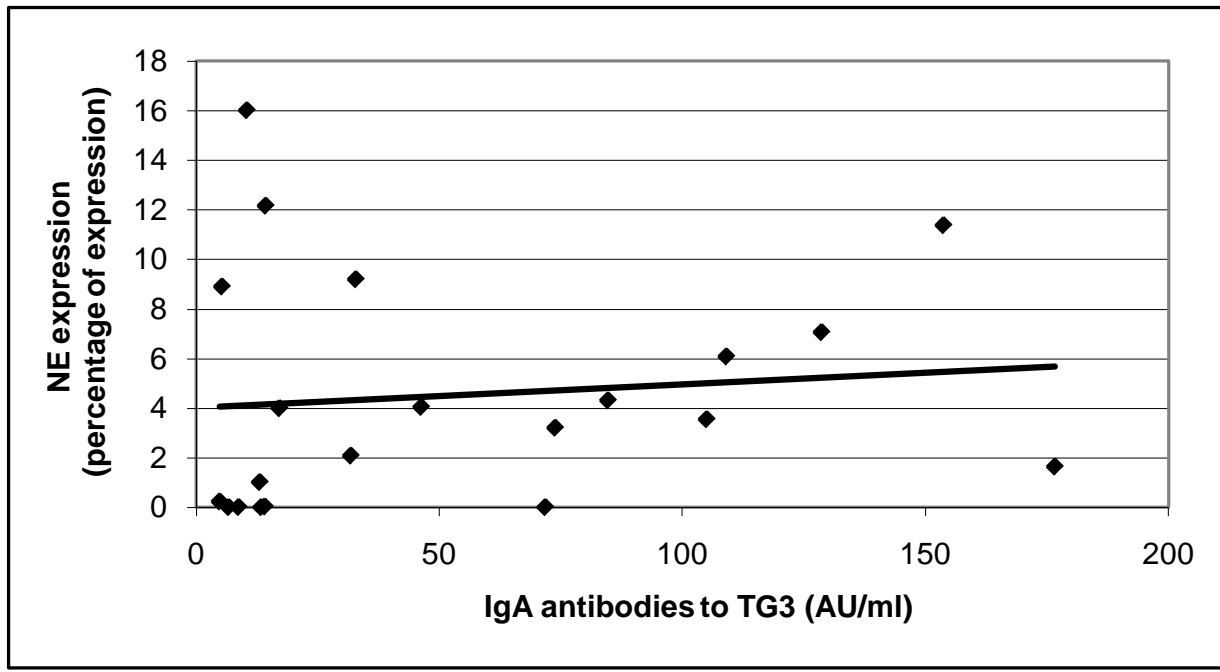

Fig. (5). Low strength of correlation between the intensity of NE expression in lesional skin and the level of serum IgA antibodies to TG3 in DH (Spearman's $r=0.2822$; $P$-value $<0.05$ ). 
response to the production of $\operatorname{IgA}$ autoantibodies to various TG3 isoforms. Alternatively, IgA autoantibodies to TG3 might activate neutrophils to releasing matrix metalloproteinases. Matrix metalloproteinases in turn might more significantly contribute to the DEJ damage in DH than in BP [14]. Yet another possibility is that other autoantigen-autoantibody systems act on cutaneous neutrophils such as $\operatorname{IgA} / \operatorname{IgA} 1$ autoantibodies to $\mathrm{TG} 2$, the autoantigen supposedly more celiac disease-specific than DH-specific. Finally, it is conceivable that the main contribution of neutrophils to the $\mathrm{DH}$ cutaneous pathophysiology might not be connected with release of DEJ-degrading enzymes. In addition to releasing them, neutrophils in DH might be primarily involved in antigen-specific (TG3 and/or TG2-driven) autoimmune response, as it has been shown that a subpopulation of human neutrophils possesses a T cell receptor (TCR)-based variable immunoreceptor [15]. It seems though that the TG3 involvement in $\mathrm{DH}$ pathogenesis might not be as profound as it has initially been suggested.

\section{REFERENCES}

[1] Sárdy M, Kárpáti S, Merkl B, et al. Epidermal transglutaminase (TGase 3) is the autoantigen of dermatitis herpetiformis. J Exp Med 2002; 195: 747-57.

[2] Shimanovich I, Mihai S, Oostingh GJ, et al. Granulocyte-derived elastase and gelatinase B are required for dermal-epidermal separation induced by autoantibodies from patients with epidermolysis bullosa acquisita and bullous pemphigoid. J Pathol 2004; 204: 51927.

[3] Leighty L, Li N, Diaz LA, Liu Z. Experimental models for the autoimmune and inflammatory blistering disease, bullous pemphigoid. Arch Dermatol Res 2007; 299: 417-22.

[4] Oikarinen AI, Zone JJ, Ahmed AR, et al. Demonstration of collagenase and elastase activities in the blister fluids from bullous skin diseases. Comparison between dermatitis herpetiformis and bullous pemphigoid. J Invest Dermatol 1983; 81: 261-6.
[5] Oikarinen AI, Reunala T, Zone JJ, Kiistala U, Uitto J. Proteolytic enzymes in blister fluids from patients with dermatitis herpetiformis. Br J Dermatol 1986; 114: 295-302.

[6] Dmochowski M, Bowszyc-Dmochowska M, Dańczak-Pazdrowska A. On patterns of IgA deposits in the skin of patients with dermatitis herpetiformis. Post Derm Alerg 2003; 20: 46-8 (in Polish).

[7] Wolnik-Trzeciak G, Bowszyc-Dmochowska M, Dańczak-Pazdrowska A, Dmochowski M. IgA antibodies to epidermal transglutaminase, tissue transglutaminase and endomysium in relation to patterns of IgA cutaneous deposits in dermatitis herpetiformis. Dermatol Klin 2005; 7: 61-5 (in Polish).

[8] Dmochowski M, Wolnik-Trzeciak G, Bowszyc-Dmochowska M, Kaczmarek E. On significance of autoimmunity to epidermal transglutaminase in human dermatitis herpetiformis. J Invest Dermatol 2006; 126 (Suppl): 5.

[9] Kaczmarek E, Strzelczyk R. From two to three-dimentional visualisation of structures in light and confocal microscopy-application for biomedical studies. In: Mendez Vilas A, Labajos-Broncano L, Eds. Current Issues on Multidisciplinary Microscopy Research and Education. Badajoz: Formatex 2005; 289-95.

[10] Bowszyc-Dmochowska M, Seraszek A, Kaczmarek E, Dmochowski M. Expression of neutrophil elastase in lesional skin is not different in patients with dermatitis herpetiformis and bullous pemphigoid. Dermatol Klin 2008; 10: 59-61 (in Polish).

[11] Munro BH. Correlation. In: Munro BH, Eds. Statistical Methods for Health Care Research. Philadelphia: Lippincott Williams \& Wilkins 2004; 239-58.

[12] Hull CM, Liddle M, Hansen N, et al. Elevation of IgA antiepidermal transglutaminase antibodies in dermatitis herpetiformis. Br J Dermatol 2008; 159: 120-4.

[13] Zocci L, Terrinoni A, Candi E, et al. Identification of transglutaminase 3 splicing isoforms. J Invest Dermatol 2007; 127: 1791-4.

[14] Salmela MT, Pender SLF, Reunala T, et al. Parallel expression of macrophage metalloelastase (MMP-12) in duodenal and skin lesions of patients with dermatitis herpetiformis. Gut 2001; 48: 496502.

[15] Puellmann K, Kaminski WE, Vogel M, et al. A variable immunoreceptor in a subpopulation of human neutrophils. Proc Natl Acad Sci USA 2006; 103: 14441-6.

(C) Bowszyc-Dmochowska et al.; Licensee Bentham Open.

This is an open access article licensed under the terms of the Creative Commons Attribution Non-Commercial License (http://creativecommons.org/licenses/ by-nc/3.0/) which permits unrestricted, non-commercial use, distribution and reproduction in any medium, provided the work is properly cited. 